\title{
Names of referees who worked on papers submitted between July 2016 and September 2017
}

The editorial board of Radioprotection, the journal of the French radiation protection society, wishes to warmly thank all the reviewers listed below who accepted to review the manuscripts published during this year. Peer review is an essential part of the process of academic publication and contributes to the quality improvement of our journal which could not exist without the commitment of our reviewers.

\author{
AMBROZOVA Iva, RÉPUBLIQUE TCHÈQUE \\ AMEON Roselyne, FRANCE \\ AMMERICH Marc, FRANCE \\ AVERBECK Dietrich, FRANCE \\ BARBIER-SALSI Annick, FRANCE \\ BEAUMONT Tiffany, FRANCE \\ BELLY-POINSIGNON Anne, FRANCE \\ BERARD Philippe, FRANCE \\ BERTHO Jean-Marc, FRANCE \\ BLANCHARDON Eric, FRANCE \\ BLANCHIN Nicolas, FRANCE \\ BOTTOLLIER-DEPOIS Jean-François, FRANCE \\ BOURGUIGNON Michel, FRANCE \\ CALVEZ Marianne, FRANCE \\ CANTARINHA Alfredo, FRANCE \\ CARREAU Edouard, FRANCE \\ CHAPON Virginie, FRANCE \\ CHAMPION Didier, FRANCE \\ CHAUMET-RIFFAUD Philippe, FRANCE \\ CHOJNACKI Eric, FRANCE \\ DAVESNE Estelle, FRANCE \\ De SAINT HUBERT Marijke, BELGIQUE \\ DESCHAMPS François, FRANCE \\ DUBLINEAU Isabelle, FRANCE \\ DUFFA Céline, FRANCE \\ EN NOURHI Karim, FRANCE \\ ETARD Cécile, FRANCE \\ ETZOL Julie, FRANCE \\ FARAH Jad, FRANCE \\ FEVRIER Laureline, FRANCE \\ FORBES Aurélie, FRANCE \\ FRANCOIS Agnès, FRANCE \\ GALLEGO Eduardo, ESPAGNE \\ GERSIN Joel, FRANCE \\ GREGOIRE Eric, FRANCE \\ GREMY Olivier, FRANCE \\ GRIFFITHS Nina, FRANCE \\ GROETZ J.E., FRANCE \\ GRUEL Gaëtan, FRANCE \\ GUIPAUD Olivier, FRANCE \\ GSCHWIND Régine, FRANCE \\ HABIB-GERYES Bouchra, FRANCE \\ HUET Christelle, FRANCE \\ JOVANOVIC Zoran, SERBIE \\ KHELIFI Rachid, ALGÉRIE \\ KIM Sang Bog, CANADA
}

Michel Bourguignon, editor in chief, Radioprotection

LAURENT Olivier, FRANCE

LEBACQ Anne-Laure, BELGIQUE

LEBARON-JACOBS Laurence, FRANCE

LEGROS Alexandre, FRANCE

LENI Pierre-Emmanuel, FRANCE

LOPEZ Maria Antonia, ESPAGNE

MAGNE Isabelle, FRANCE

MAGNIER Florian, FRANCE

MALCHAIR Francoise, BELGIQUE

MANDUCI Loredana, FRANCE

MARZOCCHI Olaf, PAYS-BAS

MATHIEU Noëlle, FRANCE

MELINTESCU Anca-Mirela, ROUMANIE

MENECHAL Philippe, FRANCE

MERCAT Catherine, FRANCE

MILOUDI Hanane, FRANCE

MURITH Christophe, SUISSE

MUSEUX Emmanuel, FRANCE

PAQUET François, FRANCE

PERRIN Anne, FRANCE

PETEGNIEF Yolande, FRANCE

PORT Mathias, ALLEMAGNE

POURCELOT Laurent, FRANCE

RAMOS DE ANDRADE Edson, BRÉSIL

RANNOU Alain, FRANCE

RENAUD Philippe, FRANCE

SAIDOU Ous, CAMEROUN

SARRAZIN Thierry, FRANCE

SAYAH Rima, FRANCE

SCHIEDTS Dominique, FRANCE

SIISKONENE Teenu, FINLANDE

SINGH Vishwanath, INDE

SOUQUES Martine, FRANCE

STAEBLER Patrick, FRANCE

STRUELENS Lara, BELGIQUE

TAMARA Radia, FRANCE

TAMPONNET Christian, FRANCE

THOMASSIN Alain, FRANCE

TIRMARCHE Margot, FRANCE

TRINKL Sebastian, ALLEMAGNE

TROMPIER François, FRANCE

VAILLANT Ludovic, FRANCE

VILLOING Daphnee, ÉTATS-UNIS

WARYN Marie Joséphine, FRANCE

ZANKL Maria, ALLEMAGNE 